\title{
A tale of two velocities: Threading vs Slicing
}

\author{
R. Gharechahi ${ }^{(a)} *$, M. Nouri-Zonoz ${ }^{(a) \dagger}$ and A. Tavanfar ${ }^{(b)} \ddagger$ \\ (a): Department of Physics, University of Tehran, \\ North Karegar Ave., Tehran 14395-547, Iran. \\ (b): International Institute of Physics, \\ Universidade Federal do Rio Grande do Norte, 59078-400 Natal-RN, Brazil.
}

\begin{abstract}
Two principal definitions of a 3-velocity assigned to a test particle following timelike trajectories in stationary spacetimes are introduced and analyzed systematically. These definitions are based on the $1+3$ (threading) and $3+1$ (slicing) spacetime decomposition formalisms and defined relative to two different sets of observers. After showing that Synge's definition of spatial distance and 3velocity are equivalent to those defined in the $1+3$ (threading) formalism, we exemplify differences between the two definitions, by calculating them for particles in circular orbits in axially symmetric stationary spacetimes. Illustrating its geometric nature, the relative linear velocity between the corresponding observers is obtained in terms of the spacetime metric components. Circular particle orbits in the Kerr spacetime, as the prototype and the most well known of stationary spacetimes, are examined with respect to these definitions to highlight their observer-dependent nature.

We also examine the Kerr-NUT spacetime in which the NUT parameter contributing to the offdiagonal terms in the metric is mainly interpreted not as a rotation parameter but as a gravitomagnetic monopole charge. Finally, in a specific astrophysical setup which includes rotating black holes, it is shown how these local definitions are related to the velocity measurements made by distant observers using spectral line shifts.
\end{abstract}

\footnotetext{
* Electronic address: r.gharechahi@ut.ac.ir

$\dagger$ Electronic address: nouri@ut.ac.ir, corresponding author

‡ Electronic address: alireza.tavanfar@cern.ch
} 


\section{INTRODUCTION AND MOTIVATION}

Today the most promising theory of gravity still remains to be Einstein's general theory of relativity which formulates gravity as spacetime geometry. After introduction of any theory, specially the revolutionary ones, an obvious and usually tedious task is to define the observable quantities and their relation to the mathematical entities introduced in the formulation of that theory. The question of what is measurable and what is not, and indeed what is the meaning of measurement itself, seems to be still one of the puzzling issues in quantum mechanics, where one should relate laboratory measurements of phenomena originated at the subatomic world to the concepts and quantities introduced in the quantum mechanical formulation of the same world. In the case of general relativity and its geometrical formulation of gravity, this amounts to finding relation between geometrical entities introduced in the theory and the observations made on the large scale world or in the presence of strong gravitational fields, where this theory is supposed to be at work. Since one is allowed to use different frames and observers attached to them, one should be cautious with another complexity which arises naturally due to the observer-dependence of the observable quantities. One of the important observable quantities that is dealt with in astrophysics and cosmology, is the 3-velocity of astrophysical objects which is measured either through astrometric observations or frequency-shifted electromagnetic signals received from those objects, the so called spectroscopic velocity. Now one may ask the question what is the relation between these observationally measured values and those defined in the mathematical formulation of the theory.

Obviously, as long as one deals with the 3-velocity in the realm of Newtonian mechanics or in special relativity, the usual flat spacetime definition is employed, the so called coordinate 3-velocity. But even in this apparently simple case, both the mathematical definitions and observational methods alike, are not immune from ambiguities arising from different effects entering the measurement process. These should be taken into account to give a consistent set of definitions and measurement procedures [1]. When it comes to the curved backgrounds, it is expected that one should deal with a much more difficult task in defining a similar concept. Obviously if in curved backgrounds we are going to borrow the same basic idea used to define the 3-velocity of a particle in Newtonian mechanics, namely spatial distances and time intervals, then one should choose a decomposition scheme to introduce 
spatial and temporal intervals in the context of general relativity.

The famous example incorporating 3 -velocities is the rotation curves of disk galaxies which present strong evidence for the existence of the so called dark matter. These are plots of the magnitude of the orbital velocity of visible matter in the galaxies (or clusters of galaxies) versus radial distance from the galactic (cluster) center. Another interesting example includes orbital velocities of particles moving in near-circular orbits around rotating black hole candidates, such as in the case of accretion disks around black holes produced by the material falling into the black hole from its companion star in black hole-star binaries. Although in the first example people mostly use the usual Newtonian definition of a 3-velocity due to the fact that in the galactic scales general relativistic effects are negligible, in the second example one has to employ GR-based calculations to account for the strong gravitational field of black holes. To assign 3 -velocities to objects in curved spacetimes by local observers, one first needs to define spatial distances and time intervals between two nearby events in the underlying curved spacetime. Indeed the main idea of any splitting formalism in GR is the introduction of spatial and temporal sections of a spacetime metric so that one could assign spatial distances and time intervals to nearby events. There are two well-known decomposition formalisms namely : I- $3+1$ (or slicing formalism) and II- $1+3$ (or threading formalism). In the more famous $3+1$ splitting, the spacetime manifold is foliated into constant-time hypersurfaces and the spacetime metric is written in terms of the so called lapse function and shift vector [2]. On the other hand in the $1+3$ formulation of spacetime decomposition the propagation of light signals between any two nearby timelike observers is employed to express the spacetime metric in terms of the so called synchronized proper time interval and spatial distance [3, 4]. This is the same decomposition formalism which is employed to introduce the so called quasi-Maxwell form of the Einstein field equations in the broader context of gravitoelectromagnetism [5]. In what follows we restrict our attention to stationary spacetimes, noting that the two formalisms coincide for static spacetimes. These two different splitting methods lead to two different definitions of 3-velocities as measured relative to two different sets of observers. Now there are two questions in order 1- Whether or not and how these two definitions are related? and 2- What are their main differences, specially when applied to test particles in astrophysically relevant cases of axially symmetric spacetimes such as the Kerr metric?. To simplify things we restrict our study to particles in circular orbits in axially symmetric stationary spacetimes, noting that most interesting 
cases including the above mentioned examples fall into this category. The outline of the paper is as follows. In sections II we introduce the $1+3$ spacetime decomposition and its definition of 3-velocity. In section III we will briefly discuss Synge's formalism of spacetime measurements and show that his measures of spatial and temporal distances, and accordingly that of 3 -velocity, are equivalent to those defined in $1+3$ formalism. In section IV we introduce the slicing formalisms of spacetime decomposition and its definition of 3-velocity. In section $\mathrm{V}$, after noting that the two definitions of the 3-velocity are defined relative to two different sets of observers, we compare them and obtain their relation. These definitions are then calculated in Kerr and Kerr-NUT spacetimes. To relate these definitions of 3-velocity to those measured by astronomers we discuss a special astrophysical setting in section VI. We summarize and discuss our results in the last section.

Notations: Following Landau and Lifshitz [3] our convention for indices is such that the Latin indices run from 0 to 3 while the Greek ones run from 1 to 3. Throughout we employ gravitational units in which $c=G=1$ and indices $T$ and $S$ stand for threading and slicing formalisms respectively.

\section{DEFINITION OF 3-VELOCITY IN $1+3$ SPLITTING (THREADING) FOR- MALISM}

The $1+3$ formulation of spacetime decomposition is the decomposition of spacetime by the so called fundamental observers in a gravitational field. These observers are at fixed spatial points in the space defined by the hypersurfaces $x^{0}=$ constant, so that their timelike world lines, acting as the time lines, decompose the underlying spacetime into timelike threads (hence the name threading is justified) [3, 6, 7]. In stationary, asymptotically flat spacetimes, these observers are at rest with respect to distant observers in the asymptotically flat region [23]. In this formalism it is the light signal propagation which is employed to characterize element of spatial distances between two nearby events [3]. The spacetime metric in this formalism is expressed in the following general form:

$$
d s^{2}=d \tau_{\text {syn }}^{2}-d l_{T}^{2}=g_{00}\left(d x^{0}-g_{\alpha} d x^{\alpha}\right)^{2}-\gamma_{T_{\alpha \beta}} d x^{\alpha} d x^{\beta}
$$

where $g_{\alpha}=-\frac{g_{0 \alpha}}{g_{00}}$ and

$$
\gamma_{T \alpha \beta}=-g_{\alpha \beta}+\frac{g_{0 \alpha} g_{0 \beta}}{g_{00}} ; \gamma_{T}^{\alpha \beta}=-g^{\alpha \beta}
$$




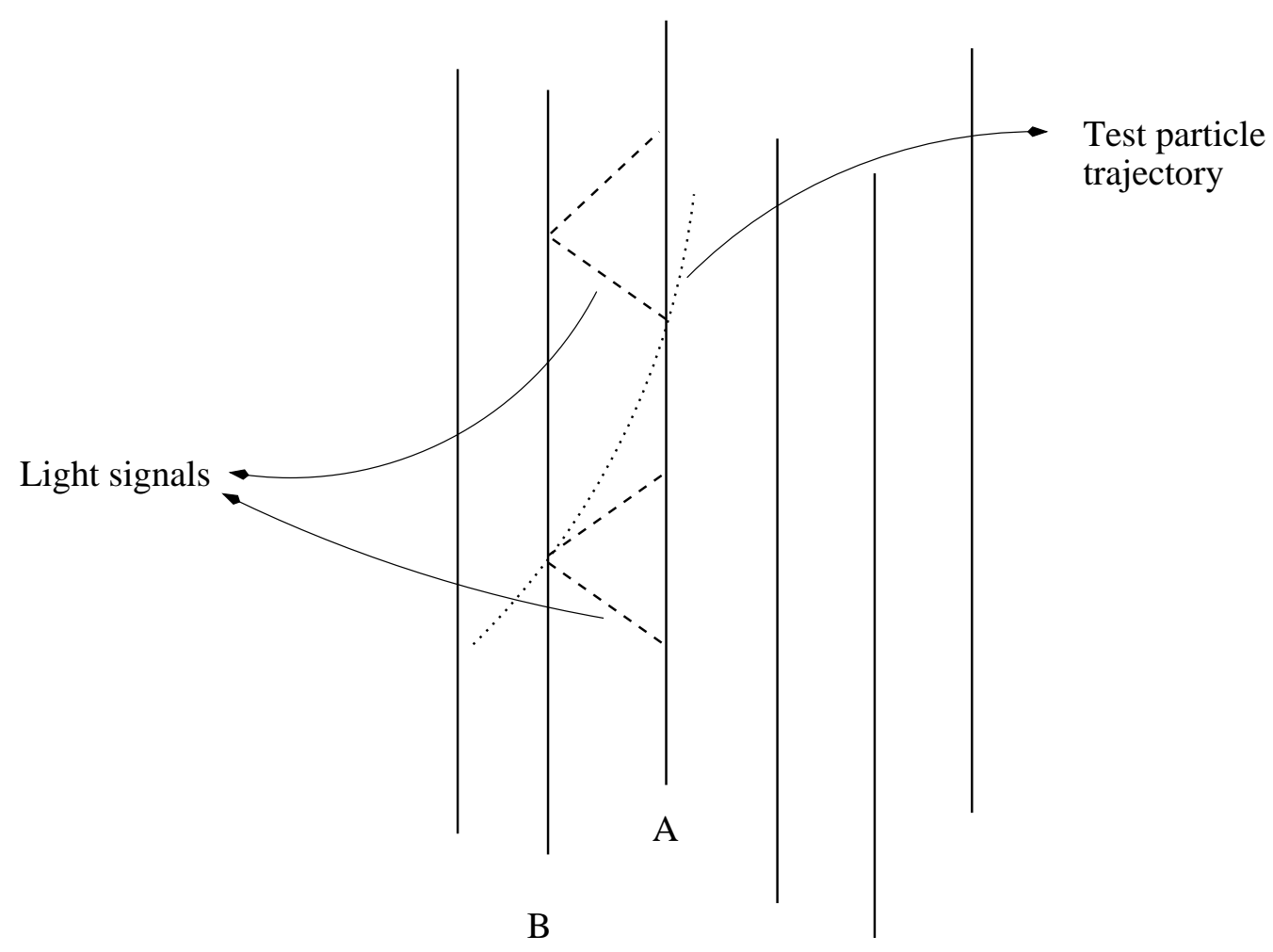

FIG. 1: A congruence of nearby worldlines of fundamental observers and a test particle crossing two of them (A and B) in the threading formulation of spacetime decomposition. Particle 3-velocity is defined in terms of the proper time read by clocks synchronized along the particle's worldline by sending and receiving light signals.

is the spatial metric of a 3 -space $\Sigma_{3}$, on which $d l_{T}$ gives the element of spatial distance between any two nearby events. The 3 -space $\Sigma_{3}$ defined in this way is called a quotient space/manifold and in general is not a submanifold of the original 4-d manifold [10, 11]. Also $d \tau_{s y n}=\sqrt{g_{00}}\left(d x^{0}-g_{\alpha} d x^{\alpha}\right)$ gives the infinitesimal interval of the so called synchronized proper time between any two events. In other words any two simultaneous events have a world-time difference of $d x^{0}=g_{\alpha} d x^{\alpha}$. In the threading formalism, the 3-velocity for a particle is defined in terms of the synchronized proper time read by clocks synchronized along the particle's trajectory. The origin of this definition of a time interval could be explained through the following procedure. If the particle departs from point B (with spatial coordinates $x^{\alpha}$ ) at the moment of world time $x^{0}$ and arrives at the infinitesimally distant point A (with spatial coordinates $x^{\alpha}+d x^{\alpha}$ ) at the moment $x^{0}+d x^{0}$, then to determine the velocity we must now take, difference between $x^{0}+d x^{0}$ and the moment $x^{0}-\frac{g_{0 \alpha}}{g_{00}} d x^{\alpha}$ which is 
simultaneous at the point B with the moment $x^{0}$ at the point A (Fig. 1). This time difference amounts to the synchronized proper time between the two nearby events (departure of the particle from point B and its arrival at point A) and upon dividing the infinitesimal spatial coordinate interval $d x^{\alpha}$ by this time difference the 3 -velocity of a particle in the underlying spacetime is given by [3]

$$
\left(v_{T}\right)^{\alpha}=\frac{d x^{\alpha}}{d \tau_{\text {syn }}}=\frac{c d x^{\alpha}}{\sqrt{g_{00}}\left(d x^{0}-g_{\alpha} d x^{\alpha}\right)}
$$

Obviously in the case of flat spacetime (i.e when $g_{00}=1$ and $g_{0 \alpha}=0$ [24]) the above definition reduces to the coordinate time velocity defined by $v^{\alpha}=\frac{d x^{\alpha}}{d x^{0}}$. One of the main features of the $1+3$ formulation is that one could express the Einstein field equations in the so called quasi-Maxwell form in the context of gravitoelectromagnetism. Using the $1+3$ formalism, it is shown that a test particle moving on the geodesics of a stationary spacetime, depart from the geodesics of the 3 -space $\Sigma_{3}$ as if acted on by the following gravitoelectromagnetic Lorentz-type 3-force [3, 5],

$$
\mathbf{f}_{g}=\frac{m_{0}}{\sqrt{1-v^{2}}}\left(\mathbf{E}_{g}+\mathbf{v} \times \sqrt{g_{00}} \mathbf{B}_{g}\right)
$$

in which the 3-velocity of the particle is defined by (3) and the GE and GM vector fields are defined as follows,

$$
\begin{gathered}
\mathbf{B}_{g}=\operatorname{curl}\left(\mathbf{A}_{g}\right) \\
\mathbf{E}_{g}=-\nabla \phi .
\end{gathered}
$$

Using the definition (3) the square of the 3-velocity is then given by,

$$
\left(v_{T}\right)^{2}=\left(\frac{d l_{T}}{d \tau_{\text {syn }}}\right)^{2}=\gamma_{T_{\alpha \beta}} v_{T}{ }^{\alpha} v_{T}{ }^{\beta}=\frac{\left(g_{0 \alpha} d x^{\alpha}\right)^{2}-g_{00} g_{\alpha \beta} d x^{\alpha} d x^{\beta}}{\left(g_{00} d x^{0}+g_{0 \alpha} d x^{\alpha}\right)^{2}} .
$$

For later comparison we note that using (1) and the above relation, the interval $d s$ could be expressed in terms of the velocity in the following form:

$$
d s^{2}=g_{00}\left(d x^{0}+\frac{g_{0 \alpha}}{g_{00}} d x^{\alpha}\right)^{2}\left[1-v_{T}^{2}\right]=d \tau_{\text {syn. }}^{2}\left(1-v_{T}^{2}\right)
$$

Restricting our attention to the case of axially symmetric stationary spacetimes in cylindrical coordinates $(t, r, z, \phi)$ with the following general form,

$$
d s^{2}=g_{t t}(r, z)(d t)^{2}+2 g_{t \phi}(r, z) d t d \phi+g_{\alpha \beta}(r, z) d x^{\alpha} d x^{\beta} \quad ; \quad \alpha, \beta=r, z, \phi
$$


we also confine the motion of the particle to a circular orbit around the axis on the $z=$ constant hypersurface such that the only non-vanishing component of the 3 -velocity has the following form

$$
\left(v_{T}^{\phi}\right)^{2}=\frac{g_{t \phi}^{2}-g_{t t} g_{\phi \phi}}{\left(g_{t t}+g_{t \phi} \omega\right)^{2}} \omega^{2} .
$$

where $\omega=\frac{d \phi}{d t}$ is the coordinate angular velocity of the particle. In the case of flat spacetime (in cylindrical coordinates) it reduces to the expected result $v_{T}^{2}=-g_{\phi \phi}\left(\frac{d \phi}{d t}\right)^{2} \equiv r^{2} \omega^{2}$.

\section{A NOTE ON SYNGE'S DEFINITION OF SPATIAL DISTANCE AND REL- ATIVE VELOCITY}

Here we digress to discuss Synge's formalism of spacetime measurements and show that it is equivalent to the $1+3$ formalism discussed above. Synge [8] in his very conceptual approach to the space and time measures, defines the spatial distance between an observer and a nearby particle as the length of the spacelike geodesic connecting them orthogonally at the position of the observer on its timelike worldline. The relative (recession) velocity of the particle with respect to the observer is defined by following the particle on its worldline at two successive points as the ratio of the difference between the lengths of the spacelike geodesics connecting the particle and the observer to the proper time difference for particle positions as measured on the observer's worldline. De Felice and Clark [9] generalized Synge's definition of spatial distance, and accordingly his definition of relative velocity, to include the case of finite distance between the observer and the particle where the spacetime curvature is not negligible and should be taken into account. For an observer with a timelike geodesic worldline $\gamma_{o}$ FIG.2 and a particle with worldline $\gamma_{p}$ at point $p_{1}$ (the event), they arrive at the following result [25],

$$
\delta T_{\gamma_{o}}\left(A_{1}, A_{2}\right)=2 L\left(p_{1}, \gamma_{o}\right)-\frac{1}{3}\left(R_{i j k l} u^{i} \xi^{j} u^{k} \xi^{l}\right) L^{3}\left(p_{1}, \gamma_{o}\right)+\mathcal{O}\left(\mid \text { Riemann }\left.\right|^{2}\right)
$$

in which $u^{i}$ and $\xi^{j}$ are the tangents to the observer worldline and the spacelike geodesic joining the observer to the particle respectively, and where $\delta T_{\gamma_{o}}\left(A_{1}, A_{2}\right)$ is the proper time difference on the observer's worldline at points $A_{1}$ and $A_{2}$ from which the observer sends and receives the light signal to the particle at its normal neighborhood. Also $L\left(p_{1}, \gamma_{o}\right)$ is the spatial distance between the observer and the particle which is taken, according to the Synge prescription, to be the length of the spacelike geodesic segment connecting them 


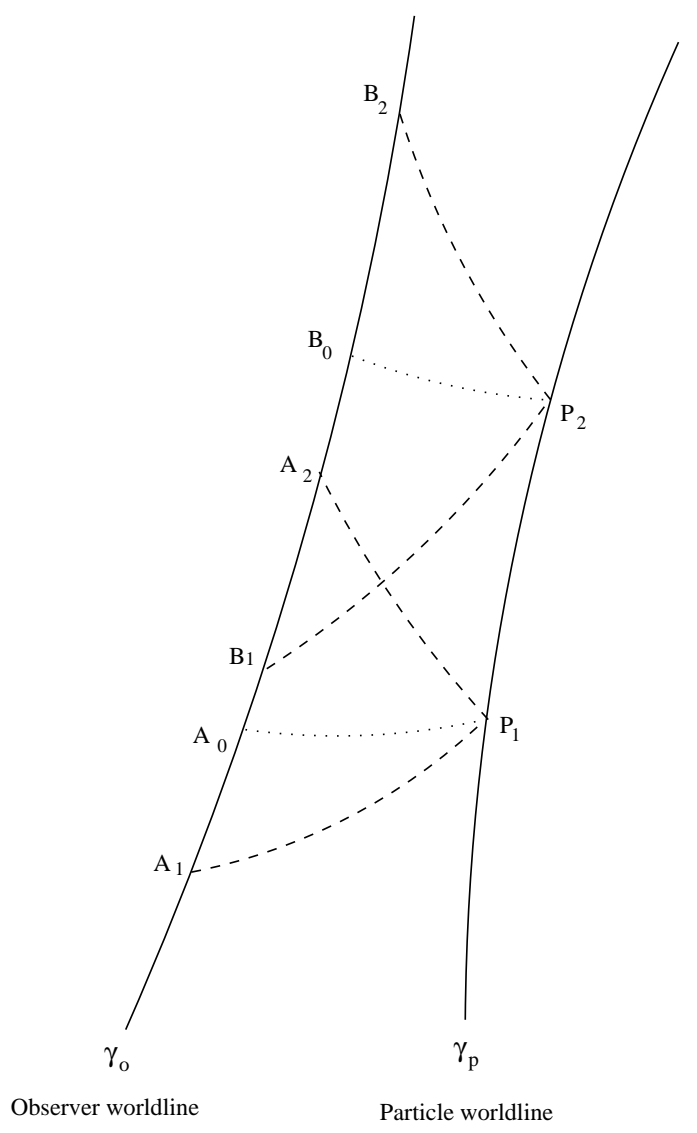

FIG. 2: The worldlines of an observer and a particle with light signals (dashed lines) sent and received by the observer to the two points $\mathrm{p}_{1}$ and $\mathrm{p}_{2}$ on the particle worldline. Also shown are the two points $A_{0}$ and $B_{0}$ which are simultaneous with these two points respectively, lying on spacelike curves (dotted lines).

orthogonally at point $A_{0}$ (i.e $\left.u^{i} \xi_{i}\right|_{A_{0}}=0$ ) on the observer's worldline between the points $A_{1}$ and $A_{2}$. Using the same measure for the spatial distance, particle's radial (recessional) velocity with respect to the observer is given by,

$$
V_{r e l}=\lim _{p_{2} \rightarrow p_{1}} \frac{\delta L}{\delta_{T_{\gamma_{o}}}}
$$

Obviously in the limit where the two worldlines are so close that the spacetime curvature could be neglected one arrives at,

$$
L\left(p_{1}, \gamma_{o}\right) \approx \frac{\delta T_{\gamma_{o}}\left(A_{1}, A_{2}\right)}{2}
$$

At the same limit, as shown by de Felice and Clarck (also implicitly by Synge himself), the spatial distance and time interval between the observer and the particle at generic events $A$ 
and $p$, as measured by the observer, reduce to the following expressions,

$$
\left(\delta L_{u}(A, p)\right)^{2}=h_{i j} d x^{i} d x^{j}+\mathcal{O}\left(d x^{4}\right) \quad ; \quad\left(\delta T_{u}\left(A_{0}, A_{1}\right)\right)^{2}=\left.u_{i} u_{j} d x^{i} d x^{j}\right|_{A}+\mathcal{O}\left(d x^{4}\right)
$$

in which $h_{i j}=g_{i j}-u_{i} u_{j}$ is the so called projection tensor. One can easily show that in the case of stationary spacetimes and in a coordinate system adapted to the timelike Killing vector, in

which $u_{i} \doteq \sqrt{g_{00}}\left(1, \frac{g_{0 \alpha}}{g_{00}}\right)$ is the 4 -velocity of the Killing observers, the above equations reduce to those defined in the $1+3$ decomposition formalism (also called projection formalism [10]), namely equations (11) and (2) [11]. This could also be seen from the fact that according to the above setting, the points $A_{0}$ and $B_{0}$ (being on the joining spacelike geodesic segments) are the events on the observer worldline which are simultaneous with the events $p_{1}$ and $p_{2}$ on the particle's world line. This concept of simultaneity was the same used to define the synchronized proper time in the 1+3 decomposition formalism. Schematically, neglecting the spacetime curvature for nearby timelike observer/particles allows one to use locally straight lines to represent the observer worldlines as well as 45 degree lines representing the light signals in FIG.1 [3].

\section{DEFINITION OF 3-VELOCITY IN THE 3+1 SPLITTING (SLICING) FOR- MALISM}

In the $3+1$ splitting, the spacetime manifold is foliated into constant-time hypersurfaces $\Sigma_{t}$ and the (stationary) spacetime metric is written in terms of the so called lapse function $N\left(x^{\alpha}\right)$ and shift vector $N^{\beta}\left(x^{\alpha}\right)$ in the following form [2];

$$
d s^{2}=g_{a b} d x^{a} d x^{b}=\left(N^{2}-N^{\alpha} N_{\alpha}\right) d x^{0^{2}}-2 N_{\alpha} d x^{0} d x^{\alpha}-\gamma_{S_{\alpha \beta}} d x^{\alpha} d x^{\beta}
$$

in which

$$
\gamma_{S_{\alpha \beta}}=-g_{\alpha \beta} ; \gamma_{S}^{\alpha \beta}=-g^{\alpha \beta}+\frac{g^{0 \alpha} g^{0 \beta}}{g^{00}}
$$

are the $3+1$ counterparts of (2) and

$$
N_{\alpha}=-g_{0 \alpha} \quad ; \quad N^{2}=\frac{1}{g^{00}}
$$

are the shift vector and lapse function respectively. It is also noted that the lapse function $N$ measures proper time between two neighboring spacelike hypersurfaces (FIG.3) $d \tau^{2}=$ $N^{2}\left(x^{\alpha}\right) d x^{0^{2}}$ while the shift vector relates spatial coordinates between them

$$
d x^{\alpha}=x_{x^{0}+\delta x^{0}}^{\alpha}-x_{x^{0}}^{\alpha}=-N^{\alpha}\left(x^{\alpha}\right) d x^{0}
$$


. Now the metric could also be written in the following decomposed form

$$
d s^{2}=d \tau_{S}^{2}-d l_{S}^{2}=N^{2} d x^{0^{2}}-\gamma_{S_{\alpha \beta}}\left(d x^{\alpha}+N^{\alpha} d x^{0}\right)\left(d x^{\beta}+N^{\beta} d x^{0}\right)
$$

showing that spatial distances over the spacelike hypersurfaces $\Sigma_{t}$ are given by

$$
d l_{S}^{2}=\gamma_{S_{\alpha \beta}}\left(d x^{\alpha}+N^{\alpha} d x^{0}\right)\left(d x^{\beta}+N^{\beta} d x^{0}\right) .
$$

Also it should be noted that the indices of 3-vectors such as the shift vector $N^{\alpha}$ are raised and lowered by the same spatial metric $\gamma_{S_{\alpha \beta} \beta}$.

In this formalism for a particle with 4 -velocity $u^{a}$, its 3 -velocity $v^{\mu}$ is defined as the projection of the particle's 4-velocity onto the above mentioned spatial slices with respect to a time coordinate reference. To this end the timelike and future-directed unit vectors normal to the slicing hypersurfaces (denoted by $n^{a}$ ) are taken as the 4-velocity of the so called Eulerian observers [26], relative to whom the 3-velocity is defined as follows

$$
v_{S}^{\alpha}=\frac{d l_{S}^{\alpha}}{d \tau}
$$

in which the norm of the displacement vector $d \mathbf{l}_{S}$ measures the distance between two spatial positions of the particle on the two hypersurfaces $\Sigma_{t}$ and $\Sigma_{t+d t}$ relative to the Eulerian observer. In the same way $d \tau$ measures the proper time on the Eulerian observer's worldline corresponding to the proper time measured on the particles worldline crossing the same hypersurfaces [27]. In this way $v$ is tangent to the hypersurface $\Sigma_{t}$ (FIG.3). In other words, from the Eulerian observers' point of view, the hypersurfaces $\Sigma_{t}$, are the set of all simultaneous events [12]. One can show that the 3 -velocity, in terms of the coordinate-time velocity, could be written in the following form [12, 13]

$$
v_{S}^{\alpha}=\frac{1}{N}\left(\frac{d x^{\alpha}}{d t}+N^{\alpha}\right)
$$

and its square, in terms of the four dimensional metric components, is given by

$$
\left(v_{S}\right)^{2}=\gamma_{S_{\alpha \beta}} v_{S}^{\alpha} v_{S}^{\beta}=g_{\alpha \beta} \frac{d x^{\beta}}{d x^{0}}\left(2 g^{0 \alpha}-g^{00} \frac{d x^{\alpha}}{d x^{0}}\right)+g_{0 \beta} g^{0 \beta}
$$

in which we used (16). Indeed using (21) one can express the metric (18) in the following equivalent form

$$
d s^{2}=N^{2} d x^{0^{2}}-d l_{S}^{2}=d \tau^{2}\left(1-v_{S}^{2}\right)
$$




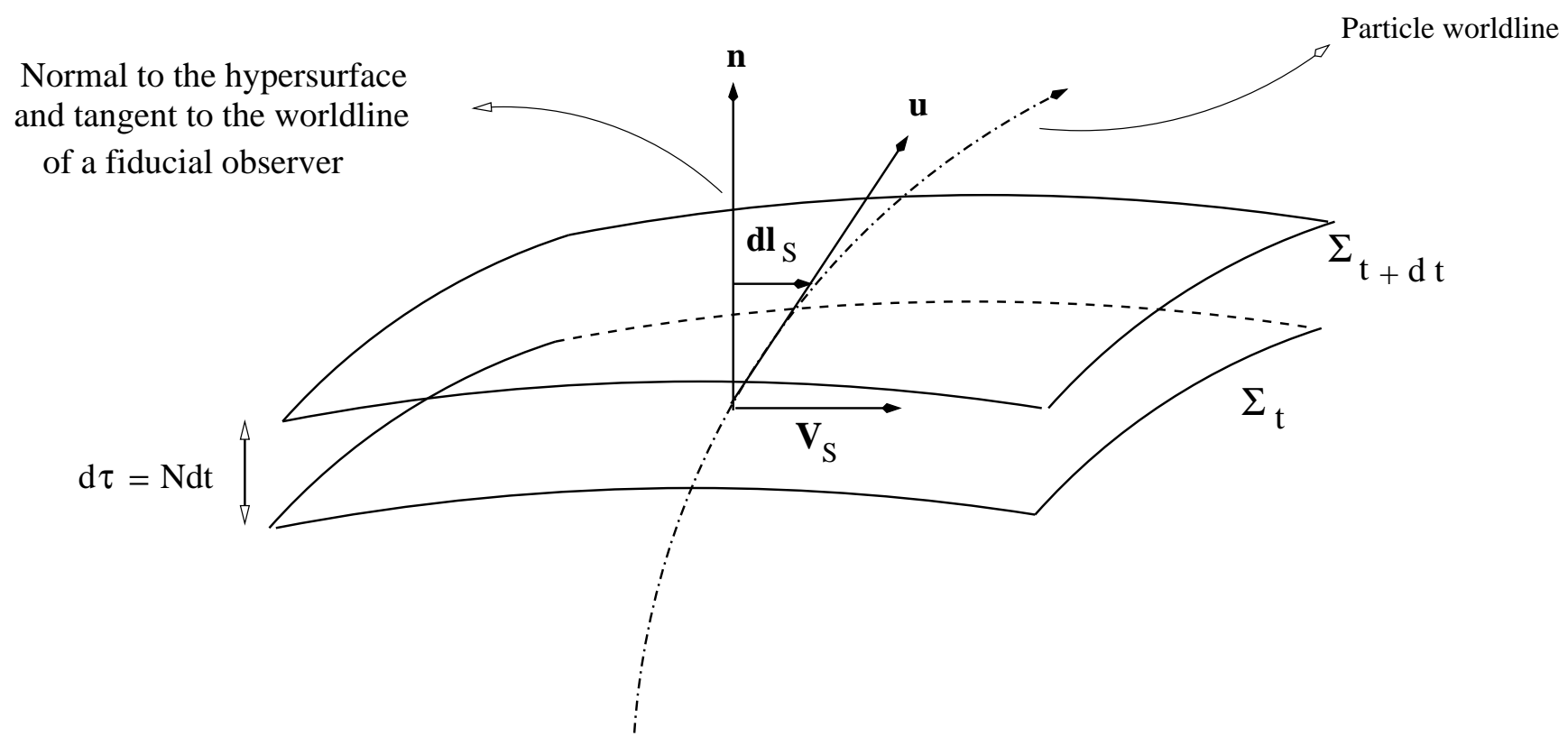

FIG. 3: Spacetime decomposition in the slicing formalism in terms of the lapse function and shift vector. Proper time and the differential element of spatial distance used in the definition of the 3 -velocity are also shown.

which is obviously the $3+1$ counterpart of (8) . Again restricting our attention to the case of axially symmetric stationary spacetimes in cylindrical coordinates, we can write the line element in its general $3+1$ decomposed form (18) as follows,

$$
d s^{2}=\left(g_{t t}-\frac{g_{t \phi}^{2}}{g_{\phi \phi}}\right) d t^{2}+g_{\phi \phi}\left(d \phi+\frac{g_{t \phi}}{g_{\phi \phi}} d t\right)^{2}+g_{r r} d r^{2}+g_{z z} d z^{2} .
$$

Restricting the motion of the particle to a circular orbit at $z=$ constant hypersurface and reading the lapse function and shift vector from the above form, the squared 3-velocity in this case is given by

$$
\left(v_{S}\right)_{\phi}^{2}=\frac{g_{\phi \phi}^{2}}{g_{t \phi}^{2}-g_{t t} g_{\phi \phi}}\left(\omega+\frac{g_{t \phi}}{g_{\phi \phi}}\right)^{2}
$$

where, as in the previous section, $\omega=\frac{d \phi}{d t}$ is the only nonzero component of the coordinate 3 -velocity. It is noted $v_{S}=0$ for $\omega=-\frac{g_{t \phi}}{g_{\phi \phi}}$ allowing the natural interpretation that $-\frac{g_{t \phi}}{g_{\phi \phi}}$ is the (dragging) angular velocity induced by the spacetime geometry [3].

Now having these two different values for the norm of the 3-velocity of a particle in circular motion in stationary, axially symmetric spacetimes we can compare them in different backgrounds. This will be done in the next section. 


\section{COMPARING THE TWO APPROACHES IN AXIALLY SYMMETRIC STA- TIONARY SPACETIMES}

To sum up the results in the last three sections, one can argue that in the threading formalism it is the fundamental observers who choose/define what should be the local space over which physical quantities are projected and measured, whereas in the slicing formalism it is the slicing subspaces (spacelike hypersurfaces) which choose/define the corresponding Eulerian observers, also called fiducial observers for the obvious reason [28]. In other words in the threading formalism fundamental objects are the observers and then, with respect to them one defines the space. But in the slicing formalism one first defines what should be the space and then according to those spatial sections defines the (fiducial) observers. This way of explaining the difference between the two formalisms actually highlights the important role of the observers in both the definition and measurement of the spatio-temporal quantities such as the 3-velocity of a particle in a gravitational field discussed in the previous sections. Indeed this is mathematically reflected in the following two metric forms in the two formalisms

$$
d s_{T}^{2}=g_{00} d x_{T}^{0^{2}}-\gamma_{T \alpha \beta} d x^{\alpha} d x^{\beta}=g_{00}\left(d x^{0}+\frac{g_{0 \alpha}}{g_{00}} d x^{\alpha}\right)^{2}+\left(g_{\alpha \beta}-\frac{g_{0 \alpha} g_{0 \beta}}{g_{00}}\right) d x^{\alpha} d x^{\beta}
$$

and

$$
d s_{S}^{2}=g_{00 S} d x^{0^{2}}-\gamma_{S \alpha \beta} d x_{S}^{\alpha} d x_{S}^{\beta}=\left(g_{00}+N^{\alpha} N_{\alpha}\right) d x^{0^{2}}+g_{\alpha \beta}\left(d x^{\alpha}+N^{\alpha} d x^{0}\right)\left(d x^{\beta}+N^{\beta} d x^{0}\right) .
$$

In the $1+3$ form the cross terms are absorbed to define a new coordinate time difference $d x_{T}^{0}=d x^{0}+\frac{g_{0 \alpha}}{g_{00}} d x^{\alpha}$ and the corresponding element of a proper time (i.e the synchronized proper time $\left.d \tau_{\text {sync }}\right)$, by using the old time-time component of the metric( i.e $\left.g_{00}\right)$. On the other hand the same terms are borrowed to define a spatial metric $\gamma_{T \alpha \beta}$ and spatial distance using the old spatial coordinate differences, namely $d x^{\alpha}$.

In the $3+1$ form the cross terms are absorbed to define a new time-time component of the metric $g_{00 S}=g_{00}+N^{\alpha} N_{\alpha}$ and the corresponding element of proper time $\left(d \tau_{S}\right)$, by using the old coordinate time difference, namely $d x^{0}$. On the other hand the same terms are also borrowed to define a new spatial coordinate difference $d x_{S}^{\alpha}=d x^{\alpha}+\frac{g^{0 \alpha}}{g^{00}} d x^{0}$ and the spatial distance using the old space-space components of the metric, namely $g_{\alpha \beta}$.

By the same token it is obvious that in the static spacetimes (i.e when $g_{0 \alpha}=0$ ) the two sets 
of observers coincide and both formalisms arrive at the same result for a particle velocity, which in the case of axially symmetric, stationary spacetimes reduce to

$$
\left(v_{S}\right)^{2}=\left(v_{T}\right)^{2}=-\frac{g_{\phi \phi}}{g_{t t}} \omega^{2} .
$$

The fact that the two definitions introduced are relative to two different sets of observers and consequently should lead to different velocity measures is more highlighted when one considers the case of a particle with $\omega \equiv \frac{d \phi}{d t}=0$, i.e a particle with zero coordinate velocity. In this case it is noted that equations (10) and (25) reduce to

$$
v_{T}=0 \quad ; \quad\left(v_{S}\right)_{\phi}^{2}=\frac{g_{t \phi}^{2}}{g_{t \phi}^{2}-g_{t t} g_{\phi \phi}}
$$

the first of which is expected intuitively since relative to a fundamental observer, by its definition, another fundamental observer's velocity (treated as a test particle with zero coordinate velocity) is zero.

On the other hand, as noted before, if we take $\omega=-\frac{g_{t \phi}}{g_{\phi \phi}}$ i.e for a particle moving with the spacetime fluid (dragged by the geometry) then

$$
v_{S}=0 \quad ; \quad\left(v_{T}\right)_{\phi}^{2}=\frac{g_{t \phi}^{2}}{g_{t \phi}^{2}-g_{t t} g_{\phi \phi}} .
$$

Again the first result is expected intuitively since a particle moving with a coordinate velocity equal to the spacetime angular velocity should be at rest relative to an (Eulerian) observer comoving with the spacetime fluid having the same angular velocity. In other words an observer attached to this particle is an Eulerian observer.

The above result is an interesting one showing that the fundamental observers and Eulerian observers which move relative to each other with the angular velocity $\omega=-\frac{g_{t \phi}}{g_{\phi \phi}}$ have the relative linear velocity whose magnitude is given by,

$$
\left(u_{T S}\right)_{\phi}=\frac{\left|g_{t \phi}\right|}{\sqrt{g_{t \phi}^{2}-g_{t t} g_{\phi \phi}}} .
$$

Since both the fundamental and Eulerian observers are local, the above assertion could be proved simply by applying the relativistic velocity addition in the following form,

$$
v_{T}=\frac{u_{T S}+v_{S}}{1+u_{T S} v_{S}}
$$

in which $v_{T}$ and $v_{S}$ are the threading and slicing 3 -velocities. It is an easy task to check that Equations (10) and (25) satisfy the above relation with $u_{T S}=-\frac{\left|g_{t \phi}\right|}{\sqrt{g_{t \phi}^{2}-g_{t t} g_{\phi \phi}}}[29]$ as 
the relative linear velocity between the two observers (frames). The above results and their interpretations become more clear when we consider explicit examples in the following subsections.

\section{A. Kerr spacetime}

Rotating stars and rotating black holes are among the most important objects in the forefront of the astrophysical observations and so any observable quantity related directly and indirectly to the rotation parameter of these objects is also of utmost importance. The spacetime metric around such sources is modeled by the Kerr metric which represents the axially symmetric, stationary spacetime around a rotating source. It has the following form in the Boyer-Lindquist coordinates,

$$
d s^{2}=\left(1-\frac{2 M r}{\rho^{2}}\right) d t^{2}+\frac{4 M a r \sin ^{2} \theta}{\rho^{2}} d t d \phi-\frac{\rho^{2}}{\Delta} d r^{2}-\rho^{2} d \theta^{2}-\left(r^{2}+a^{2}+\frac{2 M a^{2} r \sin ^{2} \theta}{\rho^{2}}\right) \sin ^{2} \theta d \phi^{2}
$$

where

$$
\rho^{2}=r^{2}+a^{2} \cos ^{2} \theta \quad, \quad \Delta=r^{2}-2 M r+a^{2}
$$

Here we restrict our attention to the orbits of particles in the equatorial plane $\left(\theta=\frac{\pi}{2}\right)$ for which the spacetime metric is given by

$$
d s^{2}\left(\theta=\frac{\pi}{2}\right)=\left(1-\frac{2 M}{r}\right) d t^{2}+\frac{4 M a}{r} d t d \phi-\left(r^{2}+a^{2}+\frac{2 M a^{2}}{r}\right) d \phi^{2}-\frac{r^{2}}{\Delta} d r^{2} .
$$

In this special case the timelike and null orbits in the Kerr geometry are described by two constants of motion which correspond to the total energy and angular momentum along the symmetry axis [15, 16]. It is also noted that in this case we are allowed to use formulas obtained in previous sections in cylindrical coordinate due to the fact that the Boyer-Lindquist coordinates coincide with cylindrical coordinates in the equatorial plane. We further restrict our attention to the circular orbits which observationally constitute an important class of orbits in this spacetime specially in the study of accretion disks around rotating black holes. In the case of Kerr metric the two sets of fundamental observers and Eulerian observers are called Zero Angular Velocity Observers (or ZAVOs) and Zero Angular Momentum Observers (or ZAMOs) respectively. As shown previously these two sets of observers and their frames move relative to each other with the angular velocity $-g_{t \phi} / g_{\phi \phi}$. In this case for test particles 
on circular orbits (as timelike geodesics), the only non-vanishing component of coordinate (angular) velocity is given by [16]

$$
\Omega \equiv \frac{d \phi}{d t}=\mp \frac{M^{1 / 2}}{r^{3 / 2} \mp a M^{1 / 2}},
$$

where the upper sign refers to retrograde (counter-rotating) orbits while the lower one refers to the direct (co-rotating) orbits. This quantity is also called Keplerian angular velocity [17] for the obvious reason that for $a=0$ it reduces to $\Omega=\frac{M^{1 / 2}}{r^{3 / 2}}$ which is the Keplerian angular velocity for a test particle in a circular orbit around a central Mass $M$ in Newtonian gravity. Circular orbits exist in the region $r>r_{p h}$, with $r_{p h}$ denoting the radial coordinate of the innermost boundary of the timelike circular orbits, the so called circular photon orbit [16],

$$
r_{p h}=2 M\left\{1+\cos \left[\frac{2}{3} \arccos \left( \pm \frac{a}{M}\right)\right]\right\} .
$$

In the case of circular orbits in the equatorial plane, the only non-vanishing component of the 3 -velocity is given by $v^{\phi}$. Now in the $3+1$ splitting formalism the 3 -velocity for direct orbits (into which we will restrict our consideration in what follows), measured relative to the ZAMO has the following form

$$
v_{S}{ }^{\phi}=\frac{1}{r \sqrt{\Delta}}\left(\left(r^{3}+a^{2} r+2 M a^{2}\right) \Omega-2 M a\right) .
$$

Substituting $\Omega$ into the above equation we end up with

$$
v_{S}{ }^{\phi}=\frac{M^{1 / 2}\left(r^{2}-2 a M^{1 / 2} r^{1 / 2}+a^{2}\right)}{\sqrt{r^{2}-2 M r+a^{2}}\left(r^{3 / 2}+a M^{1 / 2}\right)}
$$

In the $1+3$ splitting formalism the 3 -velocity (in direct orbits) measured relative to the fundamental observers at rest in the Kerr geometry has the form

$$
v_{T}^{\phi}=\frac{\left(r^{2}-2 M r+a^{2}\right)^{1 / 2}}{\left(1-\frac{2 M}{r}+\frac{2 M a}{r} \Omega\right)} \Omega
$$

again with substituting the Keplerian angular velocity as the coordinate angular velocity, we have

$$
v_{T}{ }^{\phi}=\frac{\left(M r^{2}-2 M^{2} r+a^{2} M\right)^{1 / 2}}{\left(r^{3 / 2}+a M^{1 / 2}\right)\left(1-\frac{2 M}{r}+\frac{2 M^{3 / 2} a}{r^{5 / 2}+a r M^{1 / 2}}\right)}
$$

Comparing equations equations (39) and (41), the following points are noteworthy:

I-As expected, for $r \gg 1$ ( or $\frac{M}{r}, \frac{a}{r} \ll 1$ ), in both formalisms the 3-velocities reduce to the Newtonian value $v^{\phi}=r \frac{M^{1 / 2}}{r^{3 / 2}+a M^{1 / 2}} \equiv r \Omega$. 


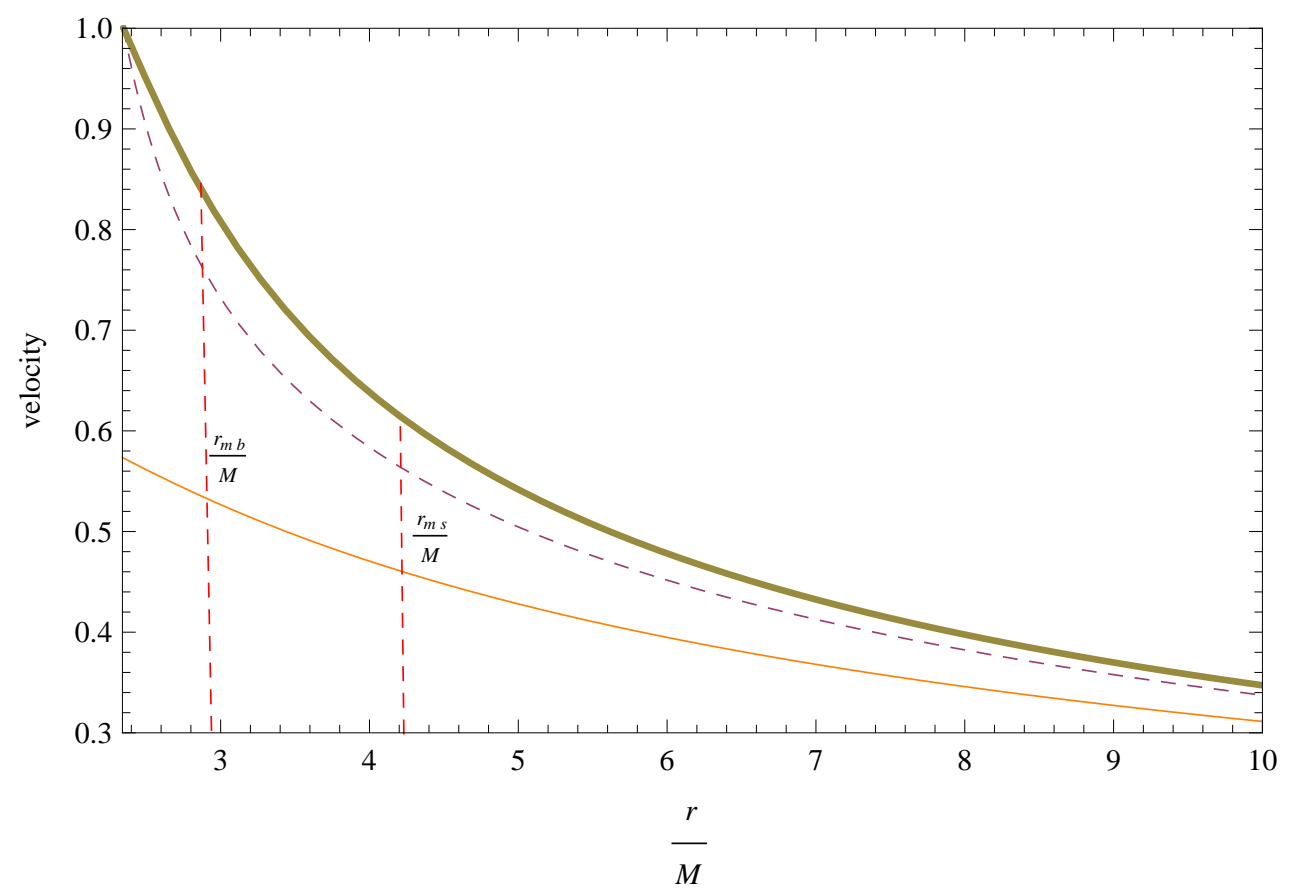

FIG. 4: Slicing 3-velocity (dashed line), threading 3-velocity (thick solid line) and coordinate 3velocity (thin solid line) of particles in circular motion in the equatorial plane of a Kerr (black) hole with $a=M / 2$. Also shown are the velocities for the normalized radial coordinates corresponding to the marginally bound $\left(r_{m b}\right)$ and marginally stable $\left(r_{m s}\right)$ circular orbits.

II-For $a=0$ both reduce to the same 3 -velocity $v^{\phi}=\frac{M^{1 / 2}}{(r-2 m)^{1 / 2}}$ corresponding to the Schwarzschild case.

III-By equation (31) the two observers move relative to each other with the following linear velocity [7]

$$
u_{T S}=\left.\frac{2 M r a \sin \theta}{\rho^{2} \Delta^{1 / 2}}\right|_{(\theta=\pi / 2)}=\frac{2 M a}{r \Delta^{1 / 2}} .
$$

In FIG.4, the above two 3-velocities along with the coordinate velocity $r \Omega$ (also could be called Keplerian orbital velocity) are depicted as functions of the normalized radial coordinate $\left(\frac{r}{M}\right)$. In both formalisms the 3 -velocity of particles in circular orbits approach the velocity of light on the photon circular orbit $\left(r_{p h}\right)$ and tend to zero at infinity.

\section{B. Kerr-NUT spacetime}

Kerr-NUT spacetime is a stationary, axially symmetric solution of Einstein vacuum field equations with three parameters $M, a$ and $n$ corresponding to mass, rotation and the NUT 
charge (also called magnetic mass) respectively. The last two parameters source the cross terms in the spacetime metric and it is naturally expected that they also contribute into the difference between the definitions of the two velocities discussed in the previous examples. This spacetime in the Schwarzschild-type coordinates has the following form

$$
d s^{2}=-\frac{\Delta}{p^{2}}(d t-A d \phi)^{2}+\frac{p^{2}}{\Delta} d r^{2}+p^{2} d \theta^{2}+\frac{1}{p^{2}} \sin ^{2} \theta(a d t-B d \phi)^{2}
$$

where

$$
\begin{array}{r}
\Delta=r^{2}-2 M r+a^{2}-n^{2}, p^{2}=r^{2}+(n+a \cos \theta)^{2} \\
B=r^{2}+a^{2}+n^{2}, \quad A=a \sin ^{2} \theta-2 n \cos \theta
\end{array}
$$

In the equatorial plane $\theta=\frac{\pi}{2}$, the line element has the form

$$
d s^{2}=-\frac{r^{2}-2 M r-n^{2}}{r^{2}+n^{2}} d t^{2}-\frac{4 a\left(M r+n^{2}\right)}{r^{2}+n^{2}} d \phi d t+\frac{B^{2}-a^{2} \Delta}{r^{2}+n^{2}} d \phi^{2}+\frac{r^{2}+n^{2}}{\Delta} d r^{2} .
$$

Circular orbits in the equatorial plane in Kerr-NUT spacetime have been studied in [18]. The angular velocity of a test particle moving on the circular orbits around the symmetry axis and is observed by stationary observers at infinity is given by

$$
\Omega=\frac{\sqrt{u^{3}\left[M\left(1-n^{2} u^{2}\right)+2 n^{2} u\right]}}{1+n^{2} u^{2} \mp a \sqrt{u^{3}\left[M\left(1-n^{2} u^{2}\right)+2 n^{2} u\right]}}
$$

where $u=\frac{1}{r}$. This formula for $n=0$ and $a=0$ gives Keplerian angular velocities in the Kerr and NUT spacetimes respectively. These circular orbits exist only for the region $r>r_{p h}$, where $r_{p h}$ is again the radius of the photon circular orbit but now given as the solution to the following equation [19]

$$
r^{3}-3 M r^{2}-3 n^{2} r \pm 2 a \sqrt{r\left(M r^{2}+2 n^{2} r-M n^{2}\right)}+M n^{2}=0
$$

Substituting the components of metric (43) in the 3 -velocity definitions in $1+3$ and $3+1$ splitting formalisms and employing the above formula for the angular coordinate velocity we have the following threading and slicing 3-velocities in the Kerr-NUT spacetime,

$$
\begin{gathered}
v_{S}^{\phi}=\frac{\mp \sqrt{u\left[M\left(1-n^{2} u^{2}\right)+2 n^{2} u\right]}\left[1+\left(a^{2}+n^{2}\right) u^{2}\right]-2 a u^{2}\left(M+n^{2} u\right)}{\sqrt{1-2 M u+\left(a^{2}-n^{2}\right) u^{2}}\left[1+n^{2} u^{2} \mp a \sqrt{u^{3}\left[M\left(1-n^{2} u^{2}\right)+2 n^{2} u\right]}\right]} \\
v_{T}^{\phi}=\frac{\left(r^{2}+n^{2}\right) \sqrt{r^{2}-2 M r+a^{2}-n^{2}}\left(\frac{\sqrt{u^{3}\left[M\left(1-n^{2} u^{2}\right)+2 n^{2} u\right]}}{\left.1+n^{2} u^{2} \mp a \sqrt{u^{3}\left[M\left(1-n^{2} u^{2}\right)+2 n^{2} u\right.}\right]}\right)}{\left[\left(r^{2}-2 M r-n^{2}\right)+2 a\left(M r+n^{2}\right)\left(\frac{\sqrt{u^{3}\left[M\left(1-n^{2} u^{2}\right)+2 n^{2} u\right]}}{1+n^{2} u^{2} \mp a \sqrt{u^{3}\left[M\left(1-n^{2} u^{2}\right)+2 n^{2} u\right]}}\right)\right]} .
\end{gathered}
$$




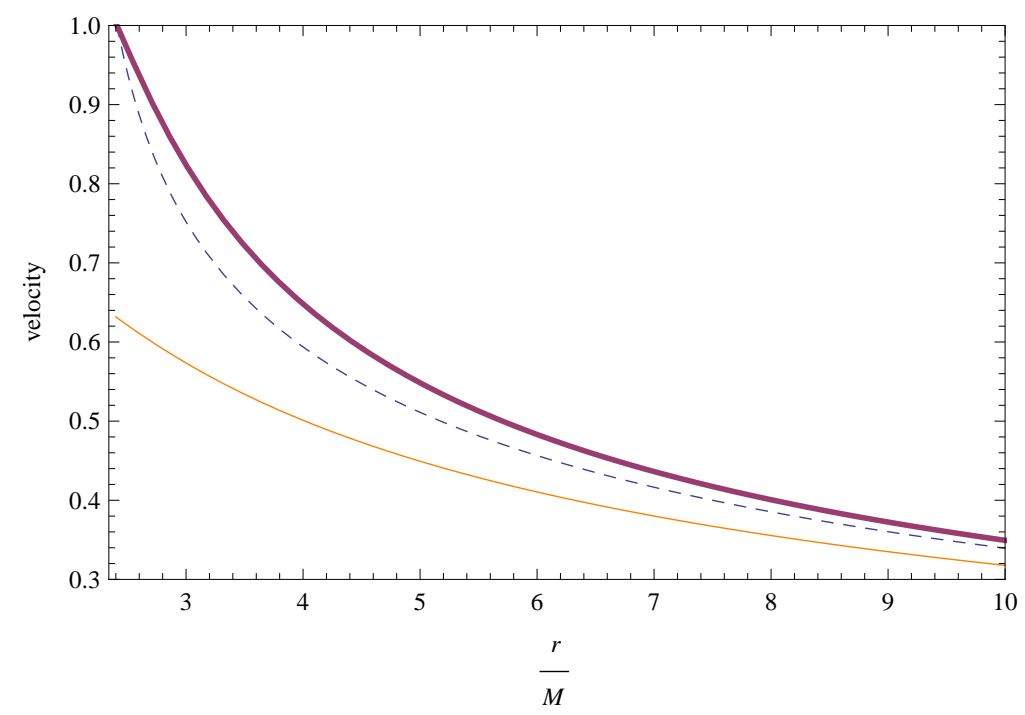

FIG. 5: Slicing 3-velocity (dashed line), threading 3-velocity (thick solid line) and coordinate 3velocity (thin solid line) of particles in circular motion in the equatorial plane of a Kerr-NUT spacetime with $a=M / 2$ and $n=M / 4$.

These are shown in FIG.5. By setting $a=0$, we recover the results for the NUT spacetime in the equatorial plane, for which the threading and slicing 3-velocities are the same given by

$$
v_{S}^{\phi}=v_{T}^{\phi}=\frac{\mp \sqrt{u\left[M\left(1-n^{2} u^{2}\right)+2 n^{2} u\right]}}{\sqrt{1-2 M u-n^{2} u^{2}}} .
$$

This is a direct consequence of the fact that the line element (43) for $a=0$ and in the equatorial plane becomes a static line element, and as pointed out earlier, in this case the two formalisms (and the two observers) coincide, leading to the same velocity definition. For any other circular orbit ( i.e with $\theta \neq \frac{\pi}{2}$ ), even in the pure NUT case (when both mass and rotation parameters are zero), the two velocities would be different. Indeed the reason that we chose the Kerr-NUT spacetime as one of our examples is the fact that although in the case of Kerr metric one could attribute the relative linear velocity between the two sets of observers to the dragging of frames (or the so called Lense-Thirring effect), that may not be so for other stationary spacetimes in which the cross terms are not necessarily originated from rotation. Indeed in the case of NUT metric, the NUT charge is mainly interpreted as representing a gravitomagnetic monopole, the gravitational analogue of the Dirac monopole [5, 20] and not a rotation parameter. So in general one could interpret this difference as a gravitomagnetic effect originated from the gravitomagnetic field of the underlying spacetime. 


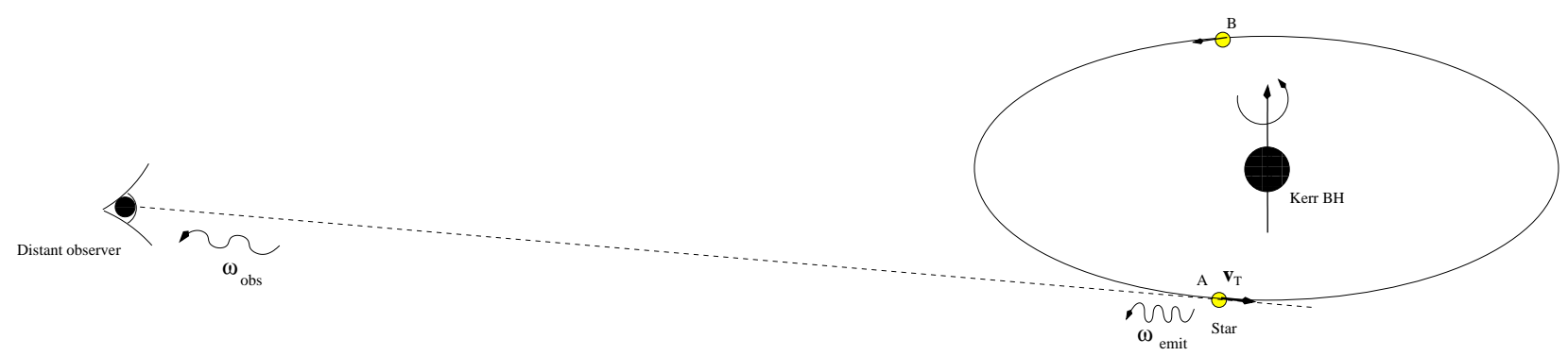

FIG. 6: A star orbiting a Kerr black hole in its equatorial plane in which also lies the line of sight of a distant observer receiving light from the star with frequency $\omega_{o b s}$.

\section{AN ASTROPHYSICAL SETUP INVOLVING LOCAL VELOCITY MEA- SUREMENT}

Having defined 3-velocity in curved backgrounds, it should be noted that these definitions of 3-velocity are given with respect to the local observers in the sense that the particle and observer are close enough for the spacetime curvature to be neglected. In the lack of real observers sitting in the strong field zone, for distant observers these are basically theoretical definitions or as Synge puts it, "Mathematical Observations" [8]. Hence one should try to relate them to the observational measurements of 3-velocity in astronomy and astrophysics which are made by distant observers at regions which may or may not be taken as asymptotically flat.

In astrophysics radial velocities are measured through the spectral line shifts of the light rays received from stars but for their transverse velocities one should use their proper motion along with their distance. Frequency shifts could be due to a combination of gravitational, cosmological as well as Doppler shifts. Here we picture an astrophysical scenario in which one could relate the emitted frequency in the rest frame of the star and that observed by a distant observer, through the frequency measured by the fundamental observer. Suppose there is a binary system either a double star or a black hole-star binary, with the smaller star orbiting the much heavier rotating star/black hole around which the geometry is represented by the Kerr metric. An example could be stars orbiting the super massive black hole in the center of our own galaxy. For our purpose, and in accordance with our calculations in section $\mathrm{V}$-A, we take the star's orbit to be a circle in the Black hole's equatorial plane which in turn is looked at as an edge-on orbit by a distant observer (FIG.6). At different positions 


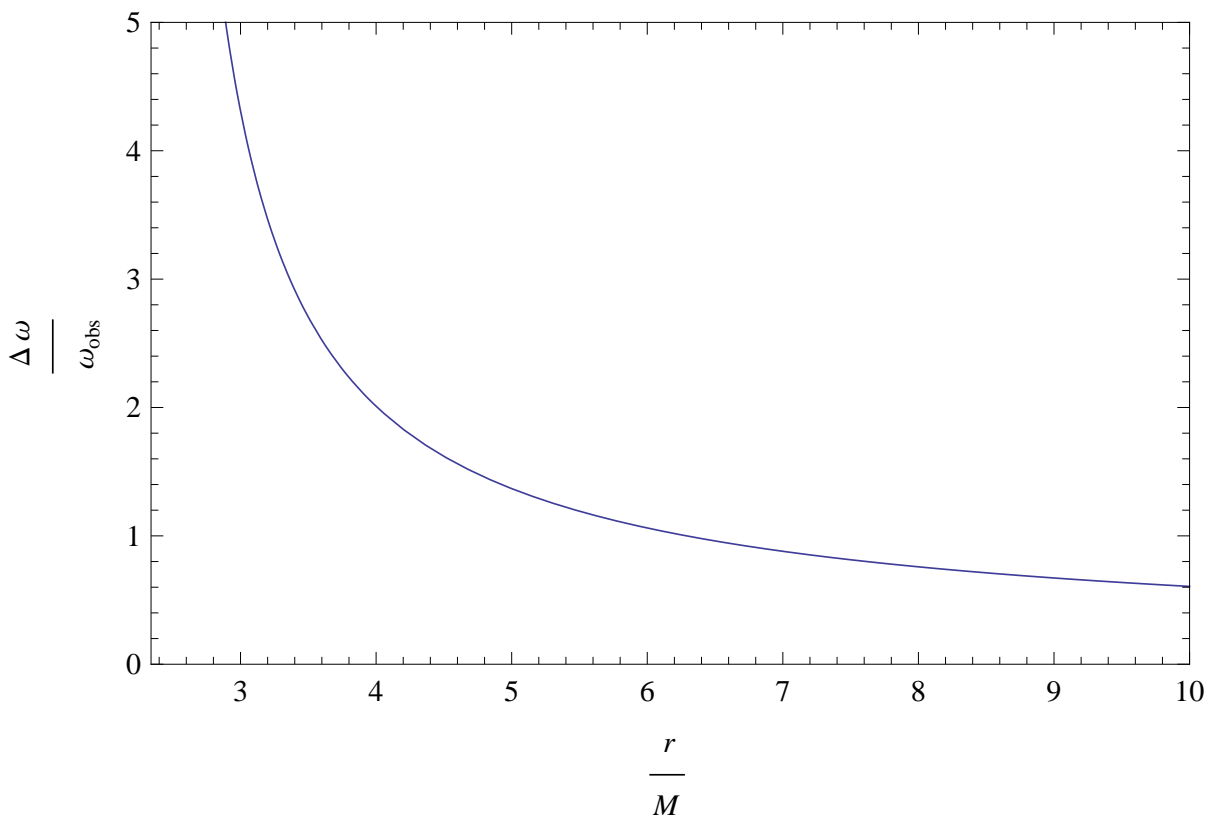

FIG. 7: Spectral shift measured by a distant observer in terms of the source's distance from the rotating black hole.

on the star's orbit the distant observer receives different frequencies, specially for the light sent from the star at points on its orbit where the observer's line of sight is tangent to the orbit, namely points $\mathrm{A}$ and $\mathrm{B}$ at which the star recedes and approaches radially both the fundamental and distant observers respectively (FIG.6). The relation between the observed and emitted frequencies could be derived in the following steps:

I-If the frequency of the light emitted by the orbiting star in its rest frame is denoted $\omega_{\text {emit }}$ and that received by the fundamental observer is given by $\omega_{f o}$, then noting that for fundamental observers the star's recessional velocity is equivalent to its Doppler velocity (as the curvature effects are taken to be negligible), we have

$$
\frac{\omega_{e m i t}}{\omega_{f o}}=\sqrt{\frac{1 \pm v_{T}}{1 \mp v_{T}}}
$$

where the upper and lower signs refer to the points A and B respectively.

II-If the frequency measured by the distant observer, residing at the asymptotically flat region, is denoted by $\omega_{\text {obs }}$ and using the fact that the fundamental observer and the star feel the same gravitational potential, the relation between the emitted and observed frequency is given by,

$$
\omega_{\text {obs }}^{A, B}=\sqrt{g_{00}\left(x_{\text {star }}^{a}\right)} \omega_{f o}=\sqrt{g_{00}\left(x_{\text {star }}^{a}\right)} \sqrt{\frac{1 \mp v_{T}}{1 \pm v_{T}}} \omega_{\text {emit }} .
$$


Substituting for the $g_{00}$ from the Kerr metric in the equatorial plane we end up with

$$
\omega_{o b s}^{A, B}=\left(\sqrt{(1-2 M / r)} \sqrt{\frac{1 \mp v_{T}(r)}{1 \pm v_{T}(r)}}\right) \omega_{\text {emit }} .
$$

The spectral shift measured by a distant observer in terms of the source's normalized radial coordinate (at point A) is shown in FIG.7. Since the gravitational redshift factor is the same everywhere on the circular orbit, the distant observer could use the frequency shift at the two points to eliminate this factor and find that

$$
\frac{\omega_{o b s}^{A}}{\omega_{o b s}^{B}}=\frac{1-v_{T}(r)}{1+v_{T}(r)} .
$$

\section{DISCUSSION}

In the present study, we considered two different definitions of 3-velocity of a test particle moving on timelike curves in a stationary spacetime. These two 3-velocities are defined in the context of the two well known spacetime decomposition formalisms, namely threading and slicing formalisms. In terms of observers, they are defined relative to two different sets of observers, fundamental observers and Eulerian observers. The two sets of observers and their corresponding definitions of 3-velocities agree when the spacetime is static but differ when the spacetime is stationary having cross terms mixing space and time coordinates. Restricting our attention to the axially symmetric stationary spacetimes which are naturally adapted to the astrophysical phenomena related mainly to rotating sources such as pulsars and Kerr black holes, we compare the two velocities as functions of radial coordinate of test particles in circular orbits around the corresponding spacetime symmetry axis. By its definition 3-velocity is an observer-dependent quantity, fundamental observers in the threading approach are at rest with respect to a rigid global coordinate system, and hence are non-inertial observers. These observers use the proper time read by clocks synchronized along the particle's worldline and employ the corresponding spatial line element $d l_{T}$ to define the 3-velocity. In the case of Kerr metric these observers are called zero angular velocity observers (ZAVOs) and it is relative to these observers that the threading velocity of a test particle is defined.

In the ADM or slicing formalism, on the other hand, the 3-velocity is defined relative to the so called fiducial observers whose worldlines are orthogonal to the slicing hypersurfaces and 
so their definition depends on the chosen slicing. In the case of stationary axially symmetric spacetimes these observers are called Eulerian observers in analogy with fluid mechanics since, although dragged by the geometry, they are at rest on spatial slices. These observers are also non-inertial and due to the fact that their 4-velocity has a vanishing rotation (by their definition), are called locally non-rotating observers [16]. In the case of Kerr metric, the same observers are called zero-angular-momentum observers (ZAMOs) [17].

It should be noted that although in the case of Kerr metric one could interpret the difference between the two velocities assigned to a particle in terms of the relative velocity between the two sets of observers originating from the dragging of frames by the Kerr hole, that would not be the case in other stationary spacetimes such as the NUT spacetime whose cross terms are not traced back to a rotation parameter. In the context of gravitoelectromagnetism one may assign this difference to the gravitomagnetic field of the underlying spacetime.

On the observational side one can think of a physical situation in which a phenomena closely related to the rotational velocity of particles near massive rotating objects is being considered such as the phenomena related to the accretion disks around rotating black holes, in which matter swallowed by the black hole from its companion star forms a rotating disk around its rotation axis. Another interesting example is the case of pulsars and related phenomena. The supermassive black hole in the center of our galaxy which harbors stars in near spherical orbits is another example in which the question of velocity measurement is an important issue. In such cases due to the high rotational velocity of the object one should treat the underlying background as a Kerr spacetime and hence two different local velocities could be assigned to the rotating matter relative to two different sets of local observers. For example in the discussion on the relation between Penrose process [21] and the energetics of superluminal jets emerging from quasars, the 3-velocity of disintegrating infalling particles are measured by ZAMO [16, 22]. As shown in Fig. 4, for particles in orbits close to the marginally bound orbit, the difference between these local velocity measures and those made by the distant observers could be as high as \%20 percent of the velocity of light. Consequently, in considering the corresponding velocity-dependent phenomena one should take into account which local velocity measure, if any, enters its formulation. 


\section{Acknowledgments}

R. Gharechahi and M. Nouri-Zonoz thank University of Tehran for supporting this project under the grants provided by the research council. M.N-Z thanks the Albert Einstein Center

for Fundamental Physics, University of Bern for supporting his visit during which this study was carried out. M.N-Z also thanks Mathias Blau for useful discussions. Alireza Tavanfar thankfully acknowledges the support of his research by the Brazilian Ministry of Science, Technology and Innovation (MCTI-Brazil).

[1] L. Lindegren and D. Dravins, Astronomy \& Astrophysics 401, 11851201 (2003).

[2] C. W. Misner, K. S. Thorne and J. A. Wheeler, Gravitation, W. H. Freeman and Company (1973).

[3] L. D. Landau and E. M. Lifishitz, Classical theory of fields, 4th Edn. Pergamon press, Oxford (1975).

[4] A. Zelmanov, Chronometric Invariants : On deformations and the curvature of accompanying space, Dissertation, Translated from the Russian and edited by Dmitri Rabounski and Stephen J. Crothers, American Research Press, 2006.

[5] D. Lynden-Bell and M. Nouri-Zonoz, Rev. Mod. Phys. 70, No. 2, 1998.

[6] D. Bini, C. Chicone, B. Mashhoon, Phys. Rev. D 85:104020, 2012.

[7] V. P. Frolov and I. D. Novikov, Black Hole Physics, Kluwer Academic Publishers (1998).

[8] J. L. Synge, Relativity: the general theory, North Holland publishing Company, Amsterdam, 1960.

[9] F. de Felice and C. J. S Clark, Relativity on curved manifolds, Cambridge University Press, 1990.

[10] H. Stephani, et. al, Exact solutions of Einsteins field equations, Cambridge University Press, 2003.

[11] M. Nouri-Zonoz and A. Parvizi, Gen. Relativ. Gravit. (2016) 48:37.

[12] K. S. Thorne and D. Macdonald, Mon. Not. R. Astron. Soc. 198, 339 (1982).

[13] E. Gourgoulhon, $3+1$ formalism and bases of numerical relativity, arXiv preprint, gr-qc/0703035 (2007). 
[14] W. Rindler, Relativity: Special, General and Cosmological, Oxford University Press, 2006.

[15] B. Carter, Phys. Rev., 174, 1559, 1968.

[16] J. M. Bardeen, W. H. Press and S. A. Teukolsky, The Astrophysical Journal, 178, 347-370 (1972).

[17] M. A. Abramowicz and P. C. Fragile, Living Rev. Relativity 16.1 (2013).

[18] C. Chakraborty, European Physical Journal C, 74(2) (2014) 1-12.

[19] P. Pradhan, Class. Quantum Grav. 32 165001, 2015.

[20] C. W. Misner, J. Math. Phys. 4, 924, 1963.

[21] R. Penrose and G. R. Floyd, Nature, 229, 177 (1971)

[22] R. M. Wald, Astrophys. J., 191, 2312341974.

[23] The space defined in this way is also called absolutely rigid space [12] or absolute space [7] and the corresponding observers/frames are alternatively called Lagrange or Killing observers/frames.

[24] Actually these are conditions for a synchronous reference system, but the only vacuum stationary spacetime in this system is the flat spacetime [3].

[25] To arrive at a simple equation, the authors consider $\gamma_{o}$ to be geodesic itself so that all the first-order curvature contribution which are coupled to the observer's acceleration vanish. This will not affect our study as we will be only concerned with the case in which the observer and particle are close enough to neglet the curvature effects.

[26] These observers are called Eulerian as they are at rest on spatial slices in the same way that in fluid mechanics the Eulerian observers are at rest in space and do not move with the fluid. To lift any ambiguities on the analogy made, it should be noted that in the case of axially symmetric stationary spacetimes these observers are co-moving with the spacetime fluid, i.e dragged by it. In other words the analogy is about the observers and their relation to the underlying 3-space and does not extend to the two fluids.

[27] By the definitions of Eulerian observers and the lapse function it is obvious that the same quantity also accounts for the proper time between two hypersurface, i.e $d \tau=N d t$.

[28] In the special case when the underlying spacetime is stationary and axially symmetric the local frames carried by these observers are also called Locally Non-Rotating Frames (LNRF).

[29] The minus sign could be traced back to the fact that for an Eulerian observer corotating with the test particle, the fundamental observer moves in the opposite sense. 